\title{
Design of Temperature Fluctuation Test Platform Based on Radiation Heat Transfer
}

\author{
Xiao Han $^{1,2}$, Ying Zhou ${ }^{2}$, Ruijin $\mathrm{Jia}^{2}$ and Zijuan Wang $^{2}$ \\ ${ }^{1}$ School of Astronautics, Beihang University, Beijing 100191, China \\ ${ }^{2}$ Beijing Institute of Spacecraft Environment Engineering, Beijing 100094, China
}

\begin{abstract}
The optical characteristics of the space science detectors are very sensitive to the temperature fluctuation. In order to study the effect of it, a method to simulate the temperature fluctuation was put forward in this paper, and the feasibility of this method was verified numerically. A radiation disturbance was artificially applied at a certain distance from the test platform, so that the surface temperature of the platform changed periodically with the radiation heater. The results show that the radiation heat transfer disturbance could produce a temperature fluctuation on the test platform, which provides a reliable theoretical support for the time-varying temperature control system.
\end{abstract}

\section{Introduction}

In recent years, gravitational wave detection technology [1] has become the focus of space science research. The LIGO [2, 3] in American, the Virgo [4] in Italy and the LISA $[5,6]$ are the largest gravitational wave detectors in the world. The gravitational waves are detected by high precision laser interferometer. However, the measurement sensitivity of laser interferometer are easily affected by the change of ambient temperature [7]. Therefore, a test platform simulating the varying temperature over time is needed to study the influence of environmental temperature on the measurement accuracy of optical instrument in ground.

Thermal alternating environment is a common test condition in environmental equipment, which is usually realized by periodically heating and cooling the controlled objects. For example, in the atmospheric thermal alternating environment test box, the circulating air is heated is heated by electric heating furnace and cooled by refrigerator. While for the space payload in vacuum environment, the air pressure is less than $1 \times 10^{-}$ ${ }^{5} \mathrm{~Pa}$, and there is no convective heat transfer. So the varying temperature of the test platform can only be realized by heat transfer of conduction and radiation.

In order to meet the special requirements of vacuum environment, a method that using a radiation heater to change the temperature of test platform was proposed in this paper. The feasibility of the method was verified through both steady state and transient calculation numerically, which provided reliable theoretical support for the realization of the subsequent temperature alternating test platform.

\section{Geometry}

The temperature test platform is shown in Fig. 1. The space science payload is placed at the center of a temperature control cabin of $600 \mathrm{~mm} \times 600 \mathrm{~mm} \times 600 \mathrm{~mm}$, where the effective test area is $300 \mathrm{~mm} \times 300 \mathrm{~mm}$ on the bottom. The whole temperature control cabin is placed in a vacuum chamber with a background temperature of $100 \mathrm{~K}$, and only radiative heat transfer is carried out with the surrounding walls, instead of convection heat transfer.

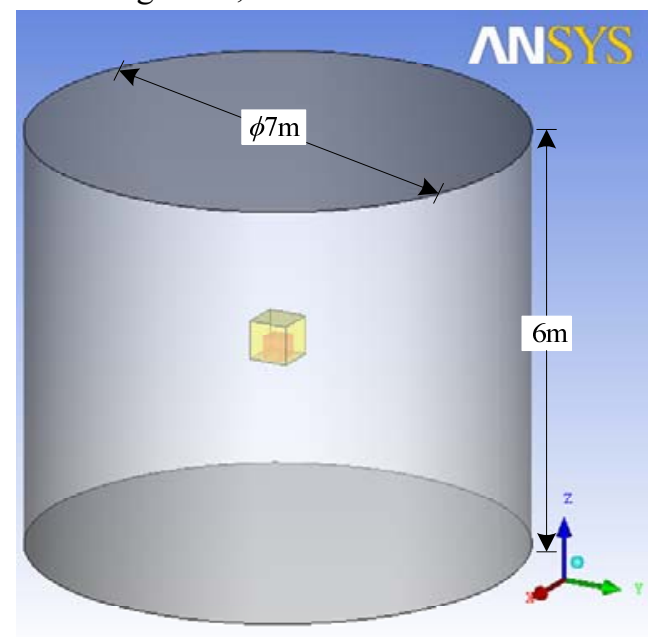

Fig. 1. Geometric model.

Fig. 2 shows the detailed structure of the temperature time-varying control system of the test platform. The temperature control cabin maintains the surface temperature on a stable base temperature $T$ by electric heating. The radiation heater heats the effective test area of the test platform to $T+\Delta T$. The temperature of the test platform changes with time through periodic work of the radiator. The radiant heater is composed of evenly 
spaced nickel-chrome metal strips, whose radiant surface is $400 \mathrm{~mm}$ from the bottom of the test platform. The metal strips are $6 \mathrm{~mm}$ wide and $0.1 \mathrm{~mm}$ thick with a spacing of $8 \mathrm{~mm}$ between each two strips.

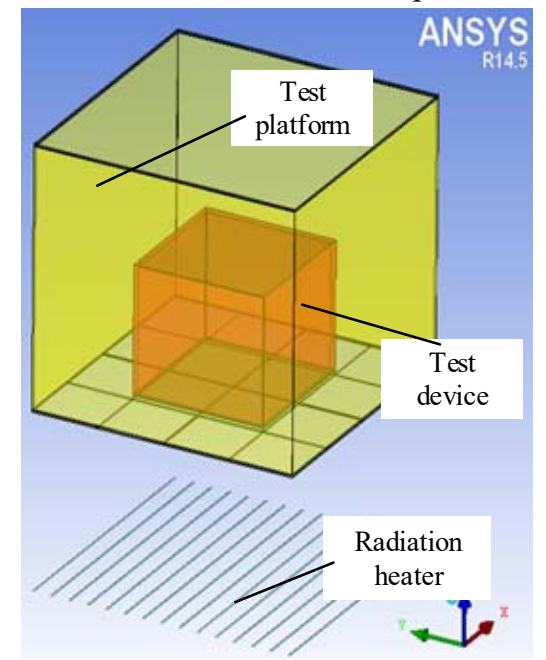

Fig. 2 Detail structure.

\section{Methodology}

The radiation heat transfer can be calculated using the formula of radiation angle factor between two dimensional parallel plates [8], as shown in Fig. 3.

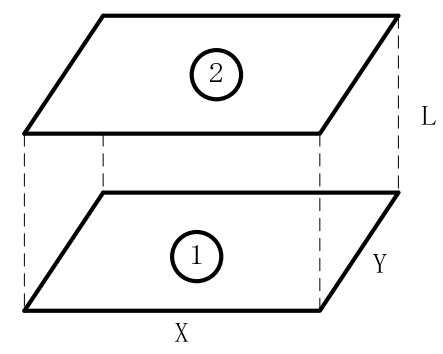

Fig. 3 Calculation model of radiation angle factor for parallel plates.

$$
\bar{X}=\frac{X}{L}, \bar{Y}=\frac{Y}{L}
$$

Name the radiation heating plate " $1 \mathrm{a}$ ", and the test platform bottom " 2 ". The radiation angle factors between the radiation heating plate $1 \mathrm{a}$ and the test platform bottom 2 can be calculated as follows:

$$
\begin{aligned}
X_{1,2}= & \frac{2}{\pi \bar{X} \bar{Y}}\left\{\ln \left[\frac{\left(1+\bar{X}^{2}\right)\left(1+\bar{Y}^{2}\right)}{1+\bar{X}^{2}+\bar{Y}^{2}}\right]^{1 / 2}\right. \\
& +\bar{X}\left(1+\bar{Y}^{2}\right)^{1 / 2} \arctan \frac{\bar{X}}{\left(1+\bar{Y}^{2}\right)^{1 / 2}} \\
& +\bar{Y}\left(1+\bar{X}^{2}\right)^{1 / 2} \arctan \frac{\bar{Y}}{\left(1+\bar{X}^{2}\right)^{1 / 2}} \\
& -\bar{X} \arctan \bar{X}-\bar{Y} \arctan \bar{Y}\}
\end{aligned}
$$

$\eta$ is a coefficient indicating the ratio of heating area to the whole area of the radiation heater.

$$
\eta=A_{1 a} / A_{1}
$$

Besides, the radiation heater will also exchange heat with the whole cold background. The angle factors are calculated by the following equation:

$$
\left\{\begin{array}{l}
X_{1 a, E u}=1-X_{1 a, 2} \\
X_{1 a, E d}=1
\end{array}\right.
$$

$X_{l a, E u}$ is the angle factors from 1a to the upper part of the background $(E u)$, and $X_{I a, E d}$ to the lower part of background $(E d)$.

The heat generated by the radiant heater 1a on the bottom of the test platform can be obtained as.

$$
\Phi_{1 a, 2}=\frac{A_{1 a}\left(E_{b 1 a}-E_{b 2}\right)}{\frac{1-\varepsilon_{1 a}}{\varepsilon_{1 a} A_{1 a}}+\frac{1}{A_{1 a} X_{1 a, 2}}+\frac{1-\varepsilon_{2}}{\varepsilon_{2} A_{2}}}
$$

According to the above equation, the temperature $T_{1 \mathrm{a}}$ required by radiation heater $1 \mathrm{a}$ can be calculated for different temperature fluctuations. Therefore, the total heating power of radiant heater 1a can be calculated as follows:

$$
\Phi_{1 a}=\Phi_{1 a, 2}+\Phi_{1 a, E u}+\Phi_{1 a, E d}
$$

The radiative heat $\Phi_{l a, E u}$ from 1 a to $E u$ is

$$
\Phi_{1 a, \mathrm{Eu}}=\frac{A_{1 a}\left(E_{b 1 a}-E_{b E}\right)}{\frac{1-\varepsilon_{1 a}}{\varepsilon_{1 a} A_{1 a}}+\frac{1}{A_{1 a} X_{1 a, \mathrm{Eu}}}+\frac{1-\varepsilon_{E}}{\varepsilon_{E} A_{E u}}}
$$

$A_{E u}$ is the area of the upper part of the background.

$$
A_{\mathrm{Eu}}=\frac{\pi D^{2}}{4}+\pi D\left(\frac{H}{2}+\frac{l}{2}+L\right)
$$

$D$ is the diameter of the chamber, $D=7 \mathrm{~m}$. And $H$ the height, $H=6 \mathrm{~m} . l$ is the edge length of the temperature testing platform, $l=0.6 \mathrm{~m}$. $L$ is the distance between the radiation heater and the bottom of platform, $L=0.4 \mathrm{~m}$.

The radiative heat $\Phi_{l a, E d}$ from 1a to Ed is as follows:

$$
\begin{aligned}
\Phi_{1 a, \mathrm{Ed}} & =\frac{A_{1 a}\left(E_{b 1 a}-E_{b E}\right)}{\frac{1-\varepsilon_{1 a}}{\varepsilon_{1 a} A_{1 a}}+\frac{1}{A_{1 a} X_{1 a, \mathrm{Ed}}}+\frac{1-\varepsilon_{E}}{\varepsilon_{E} A_{E d}}} \\
A_{\mathrm{Ed}} & =\frac{\pi D^{2}}{4}+\pi D\left(\frac{H}{2}-\frac{l}{2}-L\right)
\end{aligned}
$$

In conclusion, by adding a radiation heater between the test platform and the cold background, the temperature fluctuation on the bottom of the platform is achieved.

\section{Numerical solution and result}

Structured grids were used to mesh the computational area. The mesh is shown in Fig. 4. The operating pressure was set to $1 \times 10^{-5} \mathrm{~Pa}$. The temperature of the background was $100 \mathrm{~K}$. And the surface emissivity was 0.88 . The surface emissivity of the test platform was 0.8 . Only radiation heat transfer was considered in the calculation. The Discrete Ordinates (DO) radiation model $[9,10]$ was used. The discretization scheme of the governing equation was a two order upwind scheme, which improved the computation accuracy. The residual 
and surface temperature were monitored to determine whether the calculation was convergent. After the computation was completed, the convergence of the computation was further verified by checking the balance of the inflow and outflow energy.

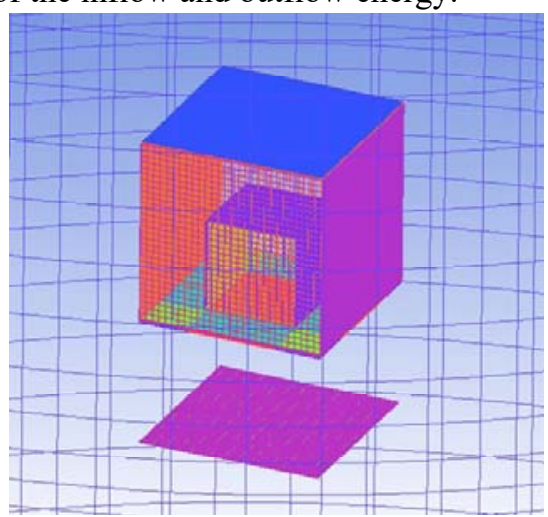

Fig. 4 Mesh for calculation.

\subsection{Steady results}

The calculation conditions include the datum temperature of the test platform at $T=-10,45^{\circ} \mathrm{C}$ and temperature fluctuation of $\Delta T=0.05,1.0^{\circ} \mathrm{C}$. As shown in Table 1.

Table 1 Parameters for steady calculation

\begin{tabular}{lccc}
\hline & $\begin{array}{c}\text { Datum } \\
\text { temperature } \\
\left({ }^{\circ} \mathrm{C}\right)\end{array}$ & $\begin{array}{c}\text { temperature } \\
\text { fluctuation } \\
\left({ }^{\circ} \mathrm{C}\right)\end{array}$ & $\begin{array}{c}\text { Radiation heater } \\
\text { power } \\
(\mathrm{W})\end{array}$ \\
\hline Case 1 & -10 & 0 & 0 \\
Case 2 & -10 & 0.05 & 1.76 \\
Case 3 & -10 & 1.0 & 13.34 \\
Case 4 & 45 & 0 & 0 \\
Case 5 & 45 & 0.05 & 2.27 \\
Case 6 & 45 & 1.0 & 24.15 \\
\hline
\end{tabular}

The surface temperature of the test area on the platform at datum temperature of $-10^{\circ} \mathrm{C}$ is shown in Fig. 5. Fig. 5 a) shows the test area temperature from $-9.92{ }^{\circ} \mathrm{C}$ to $-9.9^{\circ} \mathrm{C}$ when the radiant heater is not working (Case1). When the heater power is $1.76 \mathrm{~W}$ (Case2), the range of temperature distribution is at $-9.4^{\circ} \mathrm{C}$ to $-8.9^{\circ} \mathrm{C}$, as shown in Fig. 5 b). The surface temperature of the test platform increased by $0.05{ }^{\circ} \mathrm{C}$ under the action of the radiation heater. It can be seen from the result that the radiation heater can realize the temperature fluctuation of the test platform.



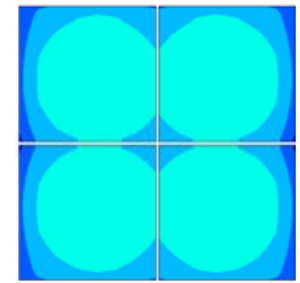

a) Case 1

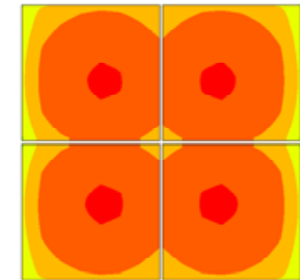

b) Case 2
Fig. 5 Surface temperature of the effective area on test platform.

As shown in Fig. 6 a), the surface temperature of the radiant heater was -118.4 to $-105.1{ }^{\circ} \mathrm{C}$ when the radiation heater was not working. It is higher than the background temperature of the shroud $\left(-173.15^{\circ} \mathrm{C}\right)$, and lower than the temperature of the test platform $\left(-10.3 \mathrm{C} \sim-9.9^{\circ} \mathrm{C}\right)$. This difference in temperature indicates that the heat is transferred from the test platform to the radiation heater, and then from the radiator to the background.
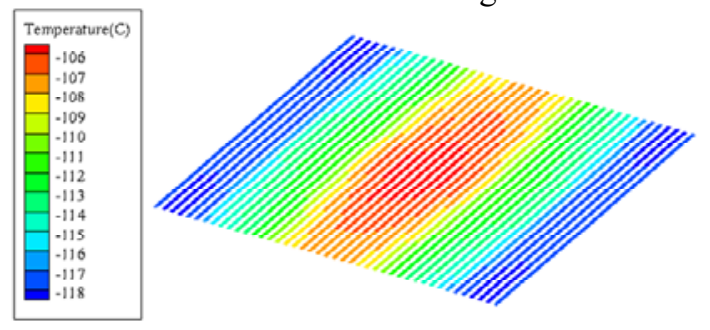

a) Case 1

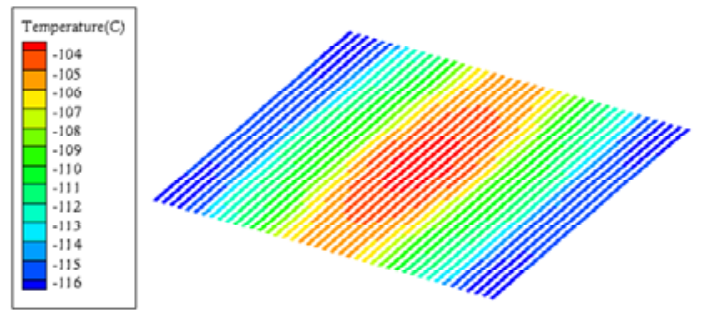

b) Case2


c) Case 3

Fig. 6 Surface temperature of radiation heater.

The surface temperature of the radiant heater at datum temperature $T=-10^{\circ} \mathrm{C}$ for fluctuation of $\Delta T=0.05$ and $1.0^{\circ} \mathrm{C}$ is shown in Fig. $6 \mathrm{~b}$ ) and c). When the radiant heater power is $P=1.76 \mathrm{~W}$, the surface temperature of the heater is $-116.5 \sim-103.6{ }^{\circ} \mathrm{C}$. When the radiant heater power is $P=13.34 \mathrm{~W}$, the surface temperature of the heater is $-87.2 \sim-72.8{ }^{\circ} \mathrm{C}$. Therefore, the surface temperature of the radiant heater under these two powers increased about $2{ }^{\circ} \mathrm{C}$ and $31{ }^{\circ} \mathrm{C}$ respectively, but the surface temperature of the test platform is only increased by $0.05{ }^{\circ} \mathrm{C}$ and $1{ }^{\circ} \mathrm{C}$. It can be seen that the disturbance exerted by the radiation heater can realize the small temperature fluctuation of the target surface by taking advantage of the large temperature change of the heater, which reduces the requirement of the ultra-high precision for the control actuator. All the results are summarized as shown in Table 2.

Table 2 Summary of steady results

\begin{tabular}{ccc}
\hline & $\begin{array}{c}\text { Surface temperature of } \\
\text { radiation heater } \\
\left({ }^{\circ} \mathrm{C}\right)\end{array}$ & $\begin{array}{c}\text { Surface temperature } \\
\text { of test platform } \\
\left({ }^{\circ} \mathrm{C}\right)\end{array}$ \\
\hline Case1 & $-118.4 \sim-105.1$ & $-9.92 \sim-9.9$ \\
\hline Case2 & $-116.5 \sim-103.6$ & $-9.87 \sim-9.85$ \\
\hline
\end{tabular}




\begin{tabular}{lcc}
\hline Case3 & $-87.2 \sim-72.8$ & $-8.95 \sim-8.9$ \\
\hline Case4 & $-96.2 \sim-74.4$ & $45.14 \sim 45.18$ \\
\hline Case5 & $-93.7 \sim-72$ & $45.2 \sim 45.23$ \\
\hline Case6 & $-57.2 \sim-37$ & $46.11 \sim 46.18$ \\
\hline
\end{tabular}

\subsection{Transient results}

When the datum temperature of the test platform was $\mathrm{T}=$ $-10{ }^{\circ} \mathrm{C}$, the dynamic process of heater's one working cycle (not heat - heat - not heat) was calculated. The curves of the average temperature of the radiation heater and the test platform with time were obtained. The heating power of the radiant heater is $1.76 \mathrm{~W}$ and $13.34 \mathrm{~W}$ respectively.

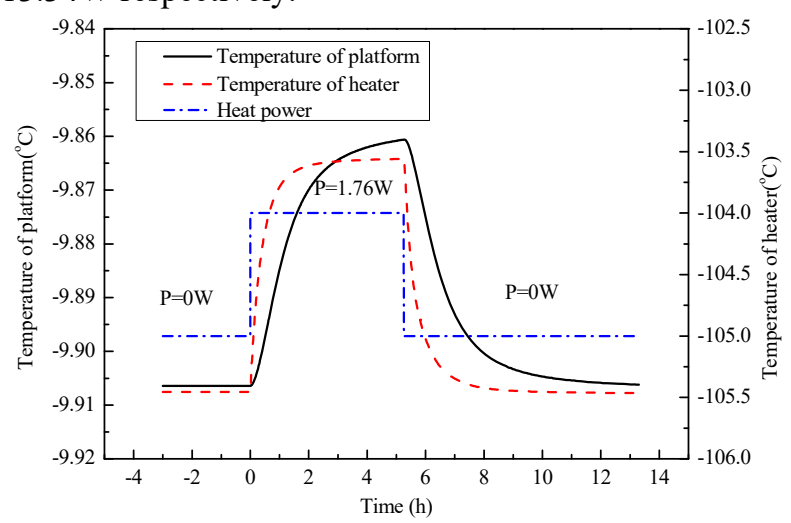

a) $P=1.76 \mathrm{~W}$

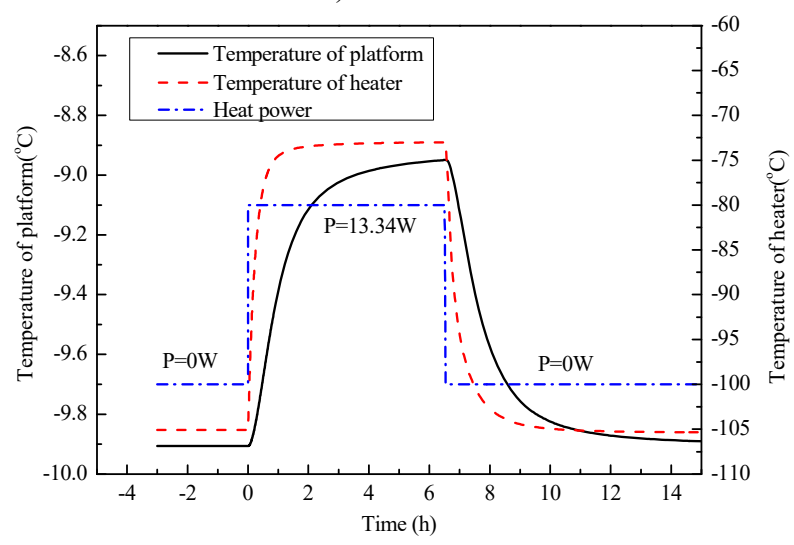

b) $P=13.34 \mathrm{~W}$

Fig. 7 Dynamic calculation results.

As shown in Fig. 7, during the heating process, the heating rate of the radiation heater is much faster than that of the test platform. When the heater is not working, both the temperature of the radiant heater and the temperature of test platform decrease rapidly, and the temperature is not overshoot. The above phenomenon is mainly caused by the temperature of the radiant heater always below the temperature of the test platform. During the whole heat transfer process, the heat is always radiated from the test platform to the heater. So the situation that the temperature of the test platform is still rising when the heater is off would never happen.

It is worth noting that it will take a long time for the test platform to heat up (more than 5 hours). This is because the heating power of the heater is always maintained at the power required for stability. Therefore, in the actual temperature control process, the test platform can reach the target temperature quickly through appropriate control methods. Since the system designed in this paper will not have temperature overshoot, it will be very beneficial to temperature control.

\section{Conclusion}

A design method of temperature alternation control on test platform surface by radiation heat transfer in vacuum environment was introduced in this paper. Through theoretical analysis and numerical simulation, the influence of the heating power of the radiation heater on the surface temperature of the test platform under the two extreme conditions $\left(-10^{\circ} \mathrm{C}\right.$ and $\left.+45^{\circ} \mathrm{C}\right)$ was studied. The temperature curves of the test platform and the radiant heater with one heating cycle were obtained through dynamic calculation. The main conclusions are as follows:

1. A design method of radiation disturbance to simulate the surface temperature fluctuation of the test platform was proposed theoretically.

2 . When the surface temperature of the test platform was increased by 0.05 and $1{ }^{\circ} \mathrm{C}$, the surface temperature of the radiant heater increased by about 2 and $31{ }^{\circ} \mathrm{C}$ respectively. Therefore, this method can achieve smaller temperature fluctuations on the target surface by using larger radiation temperature changes. Thus, the accuracy requirements of control actuators are reduced.

3. The curve of the test platform temperature with the radiant heater cycle was obtained. The results show that the test platform takes a long time to achieve the set temperature when the heating power is output at steady state. Therefore, in practical application, the power of the heater can be increased properly in the early stage, so that the temperature of the test platform can reach the set value quickly.

4. The next step is to experimentally verify the effectiveness of the present method, and to summarize the appropriate temperature control method, so that the test platform can realize temperature alternating control quickly.

\section{References}

1. B Sathyaprakash et al. Scientific objectives of Einstein Telescope. Classical and quantum gravity. 29:1-16, (2012).

2. A Abramovici et al. LIGO: The Laser Interferometer Gravitational-Wave Observatory. Science. 256: 325-333, (1992).

3. A Abramovici et al. Improved sensitivity in a gravitational wave interferometer and implications for LIGO. Physics letters A. 218: 157-163, (1996).

4. B Caron et al. A simulation program for the VIRGO experiment. Nuclear instruments and methods in physics research Section A. 360: 375-378, (1995). 
5. K Danzmann. LISA - an ESA cornerstone mission for a gravitational wave observatory. Classical and quantum gravity. 14:1399-1404, (1997).

6. P McNamara, S Vitale and K Danzmann. LISA Pathfinder. Classical and quantum gravity. 25:1-8, (2008).

7. Rowan S, Hough J. Gravitational Wave Detection by Interferometry (Ground and Space). Albert Einstein Institute, Germany: the Max Planck Institute for Gravitational Physics, 1 41, (2000).
8. Siegel R, Howell J O. Thermal radiation heat transfer. (Washington D C: Hemisphere Publishing Corporation, 1982).

9. E. H. Chui and G. D. Raithby. Computation of Radiant Heat Transfer on a Non-Orthogonal Mesh Using the Finite-Volume Method. Numerical Heat Transfer, Part B, 23:269-288, (1993).

10. S. R. Mathur and J. Y. Murthy. Coupled ordinates method for multigrid acceleration of radiation calculations. J. of Thermophysics and Heat Transfer, 13(4):467-473, (1999). 